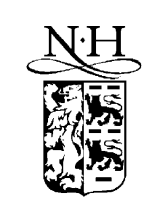

ELSEVIER

Computational Geometry 16 (2000) 35-52

Computational

Geometry

Theory and Applications

www. elsevier.nl/locate/comgeo

\title{
Efficient algorithms for line and curve segment intersection using restricted predicates
}

\author{
Jean-Daniel Boissonnat ${ }^{\mathrm{a}, *, 1}$, Jack Snoeyink ${ }^{\mathrm{b}, 2}$ \\ ${ }^{a}$ INRIA, BP 93, 06902 Sophia Antipolis, France \\ ${ }^{\mathrm{b}}$ University of British Columbia, Department of Computer Science, and \\ University of North Carolina, Department of Computer Science, CB 3175 Sitterson Hall, Chapel Hill, NC 27599-3175, USA \\ Communicated by M. Goodrich; received 12 January 1998; accepted 9 November 1999
}

\begin{abstract}
We consider whether restricted sets of geometric predicates support efficient algorithms to solve line and curve segment intersection problems in the plane. Our restrictions are based on the notion of algebraic degree, proposed by Preparata and others as a way to guide the search for efficient algorithms that can be implemented in more realistic computational models than the Real RAM.

Suppose that $n$ (pseudo-)segments have $k$ intersections at which they cross. We show that intersection algorithms for monotone curves that use only comparisons and above/below tests for endpoints, and intersection tests, must take at least $\Omega(n \sqrt{k})$ time. There are optimal $\mathrm{O}(n \log n+k)$ algorithms that use a higher-degree test comparing $x$ coordinates of an endpoint and intersection point; for line segments we show that this test can be simulated using $\mathrm{CCW}()$ tests with a logarithmic loss of efficiency. We also give an optimal $\mathrm{O}(n \log n+k)$ algorithms for red/blue line and pseudo-segment intersection, in which the segments are colored red and blue so that there are no red/red or blue/blue crossings. ๑ 2000 Elsevier Science B.V. All rights reserved.
\end{abstract}

\section{Introduction}

All too often, a proof that a geometric algorithm is correct for the Real RAM computational model [22] does not imply that a correct implementation will run correctly on the limited precision arithmetic of a real computer. This fact has spurred three branches of research: First, researchers have studied how to correctly and efficiently evaluate predicates used by geometric algorithms. Much recent work has been devoted to combining floating point filters and exact evaluation of predicates; exact computation is performed when the floating point filter fails to provide a certified answer, which is

\footnotetext{
* Corresponding author.

E-mail addresses: jean-daniel.boissonnat@ sophia.inria.fr (J.-D. Boissonnat), snoeyink@cs.unc.edu (J. Snoeyink).

${ }^{1}$ Research partially supported by ESPRIT IV LTR Project No 28155 (Galia).

${ }^{2}$ Research partially supported by grants from NSERC, the Killam Foundation, and CIES.
} 
usually rare. New methods have been designed for the exact evaluation of signs of determinants and arithmetic expressions [2,7,13], and various exact, adaptive arithmetics [9,23,24,26], and various floating point filters, both static and dynamic, have been experimentally tested $[6,8,15]$. Second, researchers have investigated algorithms that give approximate results with provable properties and guarantees on efficiency $[16,20,25]$. Third, researchers have considered the computational requirements of the problems themselves and developed algorithms that use "simpler" predicates. Our notion of "simpler" is found in Section 2.

It is this third branch that we follow in this paper. Following Boissonnat and Preparata [5], we study the classic problem of segment intersection: given a set of $n$ segments in the plane, report all pairs of intersecting segments. We consider this problem for sets of line segments and sets of segments of $x$-monotone curves in which any pair intersects in at most one point, at which they cross. That is, we restrict our attention to what may be called pseudo-segments. Some extensions to segments that intersect in two or more points are possible.

Forrest [14] has said "Mathematically, the problem of reporting intersecting segments is trivial. Computationally, the problem is far from easy, and may be impossible to solve reliably and consistently." This comment may seem surprising, since there is a simple $\Theta\left(n^{2}\right)$ time algorithm that is optimal in the worst case: check all pairs for intersection. If only $k$ pairs intersect, however, we might prefer an algorithm whose running time is sensitive to the output size $k$ as well as its input size $n$. A running time of $\mathrm{O}(n \log n+k)$ would be optimal, since $\Omega(k)$ time is required to write the output, and any algorithm that reports if any intersection occurs can solve element uniqueness, which takes $\Omega(n \log n)$ time [18].

An important question is whether a set of predicates allow an efficient algorithm. It is clear that the choice of predicates can affect the possibility of performing a computation: for example, if predicates are linear polynomials then it is impossible to determine whether two segments intersect- there is no algorithm for segment intersection at all. We show that efficiency is also affected. In Section 3 we establish an $\Omega(n \sqrt{k})$ lower bound for curve intersection algorithms that use predicates only to test order and orientation of curve endpoints, whether endpoints are above or below other curves, and whether two curves intersect (we do not allow predicates that order intersection points). Balaban's algorithm [3] can beat the lower bound and solve this problem optimally for curves using an additional predicate that compares orders of intersection points and endpoints. For line segments, we adapt Balaban's algorithm to achieve $\mathrm{O}\left(n \log ^{2} n+k \log n\right)$ time using only triangle orientation tests—often called $\mathrm{CCW}()$ tests.

In Section 4 we consider the special case of the red/blue curve intersection problem, which is to find the intersecting pairs in a set of curves that have been colored red and blue so that there are no red/red or blue/blue crossings. In this case, we obtain an optimal $\mathrm{O}(n \log n+k)$ algorithm for line segments and pseudo-segments by adapting the trapezoid sweep algorithm of Chan [10].

\section{Preliminaries and history of segment intersection}

In this section, we discuss the predicates that our algorithms and lower bounds will be using. 


\subsection{Algebraic degree}

We limit the computational predicates by algebraic degree. As formulated by Preparata and others [5, 19], an elementary predicate used by an algorithm is a test of the sign of a homogeneous multivariate polynomial whose arguments are a subset of the input variables; its degree is the maximum degree of its polynomial factors that are irreducible over the rationals and have non-constant sign. For example, the commonly-used orientation test for three points in the plane is an elementary predicate of degree 2 :

$$
\operatorname{CCW}(p, q, r)=\operatorname{sign} \operatorname{det}\left|\begin{array}{lll}
1 & p_{x} & p_{y} \\
1 & q_{x} & q_{y} \\
1 & r_{x} & r_{y}
\end{array}\right|=\operatorname{sign}\left(\left(q_{x}-p_{x}\right)\left(r_{y}-p_{y}\right)-\left(r_{x}-p_{x}\right)\left(q_{y}-p_{y}\right)\right) \text {. }
$$

A predicate, which is a Boolean combination of a constant number of elementary predicates, has degree equal to the maximum degree of its elementary predicates. The degree of an algorithm is the maximum degree of its predicates, and the degree of a problem is the minimum degree of any algorithm that solves the problem.

\subsection{Predicates for segment intersection}

Algorithms for computing the intersections of line segments or monotone curve segments typically use a subset of the following predicates, which are all to be found in Fig. 1.

a. $x$-order of endpoints: compare the $x$-coordinates of endpoints of two curves.

b. endpoint above/below curve: given a monotone curve segment $s$ and an endpoint $p$ whose $x$ coordinate lies between the $x$-coordinates of $s$, determine if $p$ is above or below $s$.

c. curve intersection test: determine whether two monotone curve segments intersect at all. If it is known that the curves cross an odd number of times, then intersection can be tested using the $x$-order and above/below predicates.

d. orientation $\mathrm{CCW}()$ : given three points, $p, q$ and $r$, determine if $\Delta p q r$ has a counter-clockwise orientation. This predicate is typically used to implement above/below or intersection tests for line segments.

e. $x$-order of endpoint and intersection point: compare the $x$ coordinates of an endpoint and an intersection point of two curves. This endpoint/intersection order test seems to be important for efficiency of segment intersection algorithms.

f. intersect in slab: determine if the intersection of two curves occurs in the vertical slab defined by endpoints of two curves. Directly reducible to the previous endpoint/intersection order test.

g. order of intersections on curve: given a monotone curve $s$, determine the order of particular intersection points with curves $t$ and $u$. This predicate is required in order to build arrangements of curves.

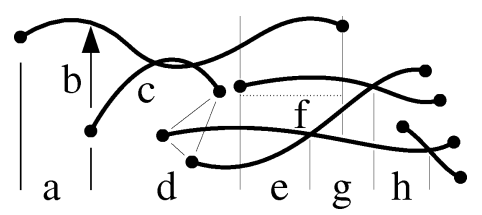

Fig. 1. Predicates a-h. 
h. $x$-order of intersections: compare the $x$-coordinates of a pair of intersection points. This predicate is required in order to build trapezoidations of curves.

\subsection{Degree of the segment intersection problem}

Boissonnat and Preparata [5] have done an extensive study of the degrees of predicates and algorithms for segment intersection in the case where line segments are specified by the coordinates of their endpoints. We summarize their findings in this subsection, then consider their extension to circular arcs as an example.

Since the intersection test can be reduced to the evaluation of degree 2 polynomials (e.g., by four orientation tests), the algorithm that checks all pairs demonstrates that the line segment intersection problem has degree 2 .

Bentley and Ottmann's sweep algorithm [4] uses the predicates for endpoint $x$-order (degree 1), endpoint/intersection order (degree 3) and intersection $x$-order (degree 5). Chazelle and Edelsbrunner's algorithm [12] avoids the last, but does use the predicate for intersection order along each segment (degree 4). These two algorithms actually solve harder problems: Bentley-Ottmann can produce a trapezoidation of the line segments, which is a degree 5 problem, and Chazelle-Edelsbrunner can produce the arrangement of segments, which is a degree 4 problem [5].

Boissonnat and Preparata [5] describe a degree 3 "lazy sweep" algorithm that uses at most the endpoint/intersection order predicate; they observe that Balaban's optimal algorithm, which we describe in detail in Section 3.2, uses the intersect in slab predicate of the same degree. Table 1 lists degrees, running times, and working space requirements (excluding output size) for these algorithms.

The general algorithms of Bentley and Ottmann, of Balaban, and of Boissonnat and Preparata can compute the intersections of monotone curves that are pseudo-segments; it suffices to adapt the same predicates to curves. When the curves may intersect in more than one point, we can still adapt the algorithms of Bentley and Ottmann (or of Balaban) to perform a linear number of additional intersection tests during the sweep to make sure that no even parity intersections are missed.

Table 1

Degrees of selected line segment intersection algorithms

\begin{tabular}{lcccc}
\hline Algorithm & Degree & $\mathrm{O}(\cdot)$ time & Space & Solves \\
\hline Check pairs & 2 & $n^{2}$ & $n$ & seg intersection \\
Bentley-Ottmann [4] & 5 & $(n+k) \log n$ & $n$ & trapezoidation \\
Chazelle-Edelsbrunner [12] & 4 & $n \log n+k$ & $n+k$ & arrangement \\
Boissonnat-Preparata [5] & 3 & $(n+k) \log n$ & $n$ & seg intersection \\
Balaban [3] & 3 & $n \log n+k$ & $n$ & seg intersection \\
This paper & 2 & $n \log ^{2} n+k \log n$ & $n$ & line seg inter. \\
\hline Boissonnat-Preparata [5] & 2 & $(n+k) \log n$ & $n$ & red/blue seg int. \\
This paper & 2 & $n \log n+k$ & $n$ & red/blue seg int. \\
\hline
\end{tabular}


Table 2

Degrees of predicates for circular arcs under three different representations

\begin{tabular}{lcccc}
\hline Predicate test & $\begin{array}{c}\text { Line } \\
\text { segments }\end{array}$ & $\begin{array}{c}\text { Semi- } \\
\text { circles }\end{array}$ & $\begin{array}{c}\text { Circle/ } \\
x \text {-range }\end{array}$ & $\begin{array}{c}\text { 3-point } \\
\text { arc }\end{array}$ \\
\hline a. $x$-order of endpoints & 1 & 1 & 1 & 1 \\
b. endpoint above/below curve & 2 & 2 & 2 & 4 \\
c. curve intersection test & 2 & 2 & 4 & 12 \\
e. $x$-order of end and intersection & 3 & 4 & 4 & 12 \\
g. order of intersections on curve & 4 & 6 & 6 & 16 \\
h. $x$-order of intersections & 5 & 12 & 12 & 44 \\
\hline
\end{tabular}

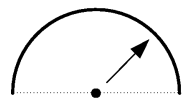

1.

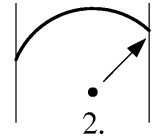

2.

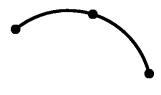

3.

Fig. 2. Representations for arcs.

As would be expected, predicates for curves have higher degree. To be more precise, we must specify the curves and their input representation. Table 2 lists bounds on the degrees of various predicates for line segments, as well as for three simple examples of representations of monotone circular arcs that are illustrated in Fig. 2.

1. semi-circles defined by center, radius, and one bit to denote upper or lower half,

2. arcs of circles defined by center, radius, the $x$ coordinates of endpoints, and one bit to denote upper or lower arc, and

3. arcs of circles defined by two endpoints and a middle point.

The proofs can be found in Appendix A.

\section{General segment intersection}

Boissonnat and Preparata [5] asked whether there is an $\mathrm{O}(n \log n+k)$-time algorithm for line segment intersection that uses only the predicates a-d. We show that for curve segments there is none by giving a lower bound of $\Omega(n \sqrt{k})$ time in Section 3.1. Then, after describing Balaban's optimal, degree 3 algorithm in Section 3.2, we show in Section 3.3 that for line segments, Balaban's algorithm can be made degree 2 with a logarithmic loss in efficiency.

Under restricted predicates, it can be helpful to consider how the input curves can be deformed without changing the results of any predicates. If an algorithm uses only the predicates for endpoint orientation and whether endpoints are above or below a curve, then the dashed curve segment in Fig. 3 is equivalent to the line segment $r$-an algorithm cannot distinguish between them.

Since the intersection point does not have a definite location when the segment $r$ is deformed, we define the witness for the intersection of $r$ and $s$ to be the leftmost endpoint $p$ that certifies that the order 


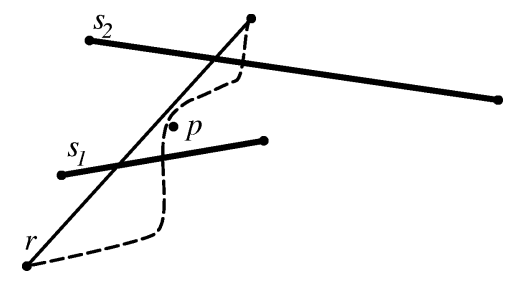

Fig. 3. Witness $p$ for $r \cap s_{1}$.

has changed from the initial order. In Fig. 3, endpoint $r$ is initially below segments $s_{1}$ and $s_{2}$, which can be checked by applying the above/below test to curves and left endpoints. Point $p$ is the witness for the intersection of $r$ with $s_{1}$, since $r$ is above and $s_{1}$ is below $p$. The right endpoint of $r$ is the witness for intersection with $s_{2}$.

Under different terminology, witnesses also play an important role in Boissonnat and Preparata's lazy sweep [5]. When a point $p$ is the next endpoint to be processed, then they call prime the pairs of consecutive segments having $p$ as witness.

\subsection{A lower bound for curves}

In this subsection, we give a lower bound for algorithms that must compute the intersection of curves from arbitrary computational tests on curve segment endpoints, tests whether an endpoint of one curve is above or below another curve, and tests that determine whether, but not where, two curves intersect (i.e., predicates a-c). Using the deformability of the curves, it is not hard to show that any such algorithm requires $\Omega(n \sqrt{k})$ tests even to count the number of intersections. (Mention of intersection tests in the following theorem is actually redundant for pseudo-segments, since we can implement the intersection test with a constant number of predicates a and b.)

Theorem 1. Any algorithm that counts all $k$ intersecting pairs among a set of $n$ segments and uses only order and orientation tests on endpoints, above/below tests for endpoints and curves, and intersection tests for curves, requires $\Omega(n \sqrt{k})$ time.

Proof. Suppose that the algorithm asks for test results from an adversary. We describe the behavior of an adversary that holds $n+m$ curves and answers in such a way that the algorithm must ask $n m$ queries to determine whether there are $\left(\begin{array}{c}m \\ 2\end{array}\right)$ or $\left(\begin{array}{c}m \\ 2\end{array}\right)+1$ intersections.

The adversary fixes all the curve endpoints so that $m$ long curve segments that all cross each other pass from left to right above $n$ shorter curve segments, as in Fig. 4. The algorithm can perform whatever computation it wishes on endpoints. For above/below or intersection tests involving two long segments, the adversary reports the order or intersection. For queries involving one or two short segments, the adversary reports "no intersection".

Because a reported intersection says nothing about the coordinates of the intersection, if the algorithm fails to ask one of the $n m$ queries about the intersection of a short segment $s$ with a long curve $\gamma$, then the adversary can deform the arrangement of long curves so that $\gamma$ has its minimum $y$ coordinate immediately above the upper endpoint of $s$. Without changing the intersection patterns or witnesses for any other curves, the adversary has the freedom to make $\gamma$ intersect $s$ or not, so the algorithm cannot have the correct number of intersections. 


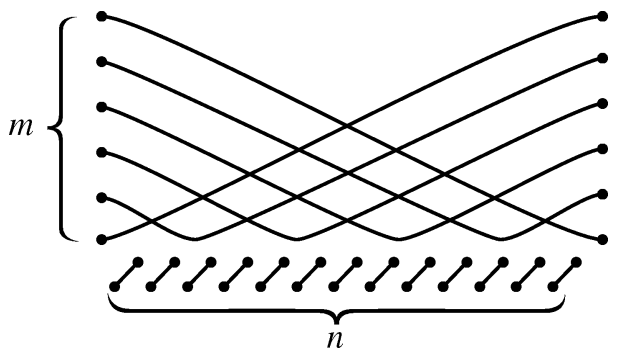

Fig. 4. The $n+m$ curves of the adversary.

Chan, in private communication, [11] has given a randomized algorithm that will run in expected $\mathrm{O}(n \sqrt{k \log n})$ time.

\subsection{Balaban's algorithm}

In 1995, Balaban gave a clever algorithm for the line segment intersection problem [3]. He first described an algorithm that runs in $\mathrm{O}((n+k) \log n)$ time and uses $\mathrm{O}(n)$ space, then used ideas from fractional cascading to remove the $\log n$ factor from the $k$. We give a high-level description of his first algorithm, and a more detailed count of the types of predicates that it uses.

Balaban applies his algorithm to a slab consisting of all points with $x$ coordinates in the half-open interval $(a, b]$, where $a$ and $b$ are $x$ coordinates of endpoints of segments. Given a set $S$ of segments that intersect the slab and whose vertical order along the line $x=a$ is known (for those segments that end to left of the slab), he computes all intersections in the slab and the order of the segments along the line $x=b$.

Segments that intersect both lines $x=a$ and $x=b$ are said to span the slab. If all the segments span the slab, then we have a portion of an arrangement of lines, and the algorithm can find the intersections and order along $x=b$ by a sorting procedure that will be given in detail in Lemma 3. Thus, we first focus on the more interesting case in which some segments end inside the slab.

A subset $A \subset S$ of the segments spanning a slab is called a staircase if no two of the segments of $A$ intersect, and $A$ is maximal-any other segment of $S$ spanning the slab intersects a segment of $A$. To find a staircase, Balaban uses a simple greedy procedure that he calls Split(). Fig. 5 illustrates segments spanning a slab, and the staircase found by Split().

Lemma 2. Given the segments $S$ that intersect a slab $(a, b]$, and the order of those that intersect the line $x=a$, a staircase can be found by $\mathrm{O}(|S|)$ endpoint/intersection order tests.

Proof. Consider the segments that span slab $(a, b]$ in order of increasing $y$ coordinate along the vertical line $x=a$. We create a staircase that contains the first segment, then repeatedly test whether the next spanning segment $s$ intersects the top segment in the staircase within the slab. If not, then add $s$ to the staircase.

It should be clear that the segments added are disjoint-if $s$ does not intersect the top segment in the slab, then it does not intersect any segment in the slab. They also form a maximal set-any segment not added intersects at least one segment in the staircase. Since we have assumed that endpoints define slab boundaries, we may use predicates that compare $x$-coordinates of endpoints to other endpoints and 


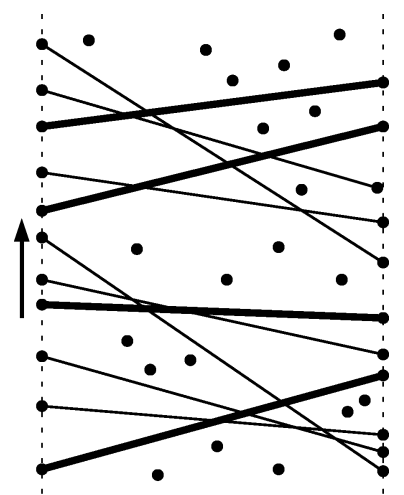

Fig. 5. Staircase found by Split().

intersection points to determine which segments span the slab and check for intersection with the top segment in $\mathrm{O}(1)$ time per segment.

Balaban's algorithm recursively finds intersecting pairs among the segments not in the staircase. It cuts the slab into two at the median $x$ coordinate of endpoints in the slab. Recursively finding the intersections in the left slab also produces the order along the cutting line. This allows the algorithm to recursively find the intersections in the right slab, which in turn gives the order of segments along the line $x=b$. The segments of the staircase can be merged into this order.

Lemma 3. Let $S$ be a set of segments intersecting slab $(a, b]$ and $S^{\prime}$ be a staircase for $(a, b]$ such that there are $k^{\prime}$ intersecting pairs with a segment from each of $S$ and $S^{\prime}$. If the order of all segments intersecting $x=a$ is known, and, in each set, the order of segments that intersect $x=b$ is known, then the intersections between $S$ and $S^{\prime}$ and the merged order along $x=b$ can be found using $\mathrm{O}\left(|S| \log \left|S^{\prime}\right|+|S|+\left|S^{\prime}\right|\right)$ above/below tests and $\mathrm{O}\left(|S|+\left|S^{\prime}\right|+k^{\prime}\right)$ endpoint/intersection order tests.

Proof. Endpoints of $S$ can be located in the staircase by binary search using above/below tests. For any segment with both endpoints in the slab, we report intersections with every staircase segment between the two endpoints. We can do the same for segments that intersect $x=a$ but not $x=b$, since we know the order along $x=a$.

For segments that intersect $x=b$, we must find the merged order and intersections. This is quite easy to do in $\mathrm{O}\left(|S|+\left|S^{\prime}\right|+k^{\prime}\right)$ operations. First, merge $S$ and $S^{\prime}$ using the ordering along $x=a$ or of endpoints in the slab. This will be the correct $y$-order along $x=b$ if there are no intersections $\left(k^{\prime}=0\right)$, and it can be checked by asking whether adjacent segments intersect in the slab using endpoint/intersection $x$-order tests. Whenever an intersection is found, it is reported, and the intersecting pair are swapped in the $y$-order and new adjacencies are tested. This produces the correct order along $x=b$ by a sort algorithm whose running time is linear in $|S|+\left|S^{\prime}\right|$ plus the number of inversions, which is the number of intersections, $k^{\prime}$.

Theorem 4. Balaban's algorithm applies $\mathrm{O}\left(n \log ^{2} n\right)$ above/below tests and $\mathrm{O}(n \log n+k)$ endpoint/ intersection order tests. 
Proof. Consider the recursion tree in which each node corresponds to a recursive call for a particular slab. The recursion tree splits the endpoints in a balanced fashion, and thus has depth $\mathrm{O}(\log n)$.

We can account for above/below tests that arise in the merge (Lemma 3 ) by charging them to endpoints. Each of the $2 n$ endpoints appears in the slab of at most one node per level, where it is charged for $\mathrm{O}(\log n)$ tests, for a total of $\mathrm{O}\left(n \log ^{2} n\right)$.

Endpoint/intersection order tests from splitting and merging (Lemmas 2 and 3) can be charged to intersections and segments. Each intersection point appears as a charge in the merge (Lemma 3) in at most one node: the nodes whose slabs contain intersection point $q$ form a path from root to leaf; the charge for $q$ is applied either at the leaf, or in the first node where one of the segments that defines $q$ joins the staircase - once a segment appears in a staircase at a node, it does not appear in a subtree of that node, so $q$ cannot be charged twice.

Each segment $s$ appears in $\mathrm{O}(\log n)$ nodes where $s$ ends in the corresponding slab, in $\mathrm{O}(\log n)$ nodes where $s$ spans the slab but does not span the parent, and in nodes where $s$ spans the slab and the parent, because it intersects a segment in the staircase of the parent. Thus, the total number of endpoint/intersection tests charged against intersections and segments is $\mathrm{O}(n \log n+k)$.

We can see that Balaban's algorithm is degree 3 for line segments; it makes heavy use of the predicate for comparing $x$ coordinates of endpoints and intersection points to determine whether intersection points occur in the slab of a node. It can apply to curves as well as line segments if we simply provide correct implementations of the predicates.

\subsection{An output-sensitive, degree-2 algorithm for segments}

The lower bound of Section 3.1 shows that Balaban's algorithm cannot be modified to find the intersections of pseudo-segments without the predicate for endpoint/intersection order. We were surprised to find that it can find the intersections of segments using only the degree-two $\operatorname{CCW}()$ test and with a logarithmic loss of efficiency. The key observation is that the lower-bound adversary can use the flexibility of the pseudo-segments to force the algorithm to explicitly obtain "no" answers to all intersection tests. With line segments, a group of "no" answers can be obtained by using CCW( ) tests to form convex hulls of endpoints and then testing tangents to the hulls. We describe this modification in this section.

We can conceptually deform the curves to push intersections to the right without crossing endpoints. This deformation preserves the witness for each intersection. Fig. 6 shows the deformation applied to the slab of Fig. 5. Notice that this is equivalent to assuming that two segments "intersect in a slab" if and only if

1. they intersect, and

2. the witness belongs to the slab.

(Since any pair of intersecting segments has a witness, two segments that intersect will intersect in some slab.) We assume that for a slab $(a, b]$ the vertical order received along $x=a$ and produced along $x=b$ are the orders of the deformed curves. We must modify the split and merge operations to respect this new order.

For Split(), we again consider the segments spanning the slab in increasing order and start by adding the first segment $s$ to the staircase; $s$ will always denote the highest segment in the staircase.

To keep track of potential witnesses in a slab, we maintain two convex hull structures: a deletiononly hull structure $\mathcal{A}$ [17] that contains all endpoints in the slab above $s$, and an insertion-only hull 


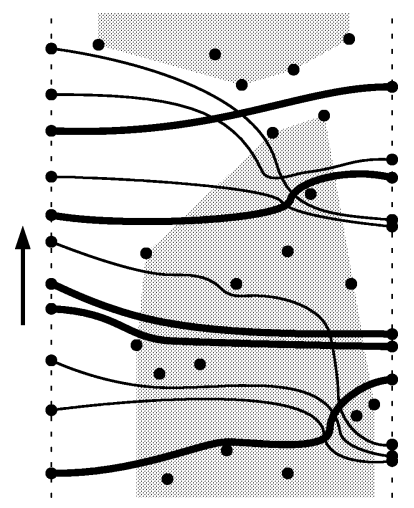

Fig. 6. Convex hulls used by Split().

structure $\mathcal{B}[22]$, that contains all endpoints below s. (It is sufficient to store the lower hull for $\mathcal{A}$ and the upper hull for $\mathcal{B}$.)

A spanning segment $t$ that enters the slab above $s$ has a witness to intersection with $s$ in the slab if and only if a point of $\mathcal{B}$ lies above $t$. It is sufficient to test one point-the vertex of the hull $\mathcal{B}$ whose tangent is parallel to $t$ - and this test point can be found on the hull in $\mathrm{O}(\log n)$ time using only $\mathrm{CCW}()$ tests.

If there is no witness, then $t$ can be added to the staircase. We remove the points below $t$ from $\mathcal{A}-$ by repeatedly deleting the vertex of $\mathcal{A}$ with tangent parallel to $t$-and insert these points into $\mathcal{B}$. Then segment $t$ becomes the new $s$. This completes the Split() operation; the final hulls are shaded in Fig. 6.

Lemma 5. Given the segments $S$ that intersect a slab $(a, b]$, and the order of those that intersect the line $x=a$, we can compute a staircase using $\mathrm{O}(|S| \log n) \operatorname{CCW}()$ tests.

We employ the convex hulls $\mathcal{A}$ and $\mathcal{B}$ again to find the intersections of the staircase with the remaining segments and determine the ordering along the line $x=b$. Notice that the convex hulls have already solved problem of locating the endpoints in the slab in the staircase. Furthermore, for segments whose right endpoint is in the slab, knowing the endpoint location and the order along the line $x=a$ is sufficient to find all intersections. We therefore assume that we have a staircase in which we have located the left endpoints of segments and we want to find their order as they cross the line $x=b$.

Recall that we cannot determine the true order, but want the order consistent with moving intersections to the right while respecting witnesses and the monotonicity condition. Fortunately, this is easier than it sounds. We use two symmetric passes to find intersections; one for segments "going up the staircase" and the second for segments "going down". To go up, build the lower hull $\mathcal{A}$ of points above the lowest segment $s$ of the staircase, and test each segment $t$ whose left endpoint is below the stair $s$ to see whether any point of $\mathcal{A}$ is also below $t$. The answer is yes if and only if $s$ and $t$ intersect in the slab; if they intersect then we can exchange their order along the line $x=b$. Thus, in $\mathrm{O}(\log n)$ time for each segment and each intersection discovered, we obtain the intersections and the order.

Lemma 6. Let $S$ be a set of segments intersecting slab $(a, b]$ and $S^{\prime}$ be a staircase for $(a, b]$ such that there are $k^{\prime}$ intersecting pairs with a segment from each of $S$ and $S^{\prime}$. If the order of all segments intersecting $x=a$ is known, and, in each set, the order of segments that intersect $x=b$ is known, 
then the intersections between $S$ and $S^{\prime}$ and the merged order along $x=b$ can be found using $\mathrm{O}\left(\left(|S|+\left|S^{\prime}\right|+k^{\prime}\right) \log n\right) \operatorname{CCW}()$ tests.

Theorem 7. Balaban's algorithm can be modified to solve the line segment intersection problem using $\mathrm{O}\left(n \log ^{2} n+k \log n\right) \operatorname{CCW}()$ tests.

Proof. As in the proof of Theorem 4, we account for the tests from split and merges by charging them to endpoints and segments in the recursion tree. We describe only the modifications.

Each endpoint is now charged for convex hull insertion and deletion in each of the $\mathrm{O}(\log n)$ slabs that contain it. The total cost of convex hull operations is bounded by $\mathrm{O}\left(n \log ^{2} n\right)$.

Because we have replaced each of Balaban's constant-time "intersection-in-slab" test by a tangent computation, each segment and intersection is now charged $\mathrm{O}(\log n)$ instead of $\mathrm{O}(1)$. Thus, the total number of tests in the algorithm is $\mathrm{O}\left(n \log ^{2} n+k \log n\right)$.

Balaban was able to obtain an optimal algorithm by shaving a factor of $\log n$ off the charge to endpoints by using the location of endpoints in slabs to help the location in their parents. Unfortunately, our extra logarithmic factor enters also on the charges to segments; it is not clear to us how to remove it.

\section{Red/blue curve intersection}

An important special case of the segment intersection problem is the red/blue segment intersection, in which the input is given as two sets of curves, one red and one blue, such that there are no red/red or blue/blue crossings. When the curves are pseudo-segments, having at most one crossing per pair, we can obtain an optimal $\mathrm{O}(n \log n+k)$ running time by modifying Chan's trapezoid-sweep algorithm [10].

\subsection{Chan's algorithm}

Chan's algorithm for red/blue segment intersection [10] works as follows. First, compute a trapezoidation of the blue segments and the red endpoints. That is, compute the decomposition of the plane that results from extending vertical segment upwards and downwards from every red and blue endpoint to the first blue segment. This can be done in $\mathrm{O}(n \log n)$ time by a standard plane sweep that keeps track of the ordering of blue segments crossing a vertical line as it sweeps across the plane.

Next, sweep over the trapezoids, where the sweep front is the boundary between those trapezoids that are entirely to the right of the vertical line $x=x_{s}$ and those that contain some point with $x$-coordinate at most $x_{s}$ (shaded in Fig. 7). During the sweep, maintain the invariant that all red/blue intersections have been reported for red segments to the left of the sweep front, up to the first (leftmost) intersection with the sweep front. In Fig. 7, the intersections for dashed portions of the red segments have not been reported; this includes where the lowermost red segment recrosses the front.

The sweep front changes when $x_{s}$ reaches the left side of a trapezoid. We re-establish the invariant by tracing any red segment that enters this trapezoid (whether from the top, bottom, or left side) through the trapezoidation until the red segment ends or reaches the sweep front. Segments that enter or leave through the top and bottom can be charged to intersections; segments that enter at left and leave at right can be considered as a group. The endpoint/intersection order test is sufficient to determine where a segment enters or leaves a trapezoid. 


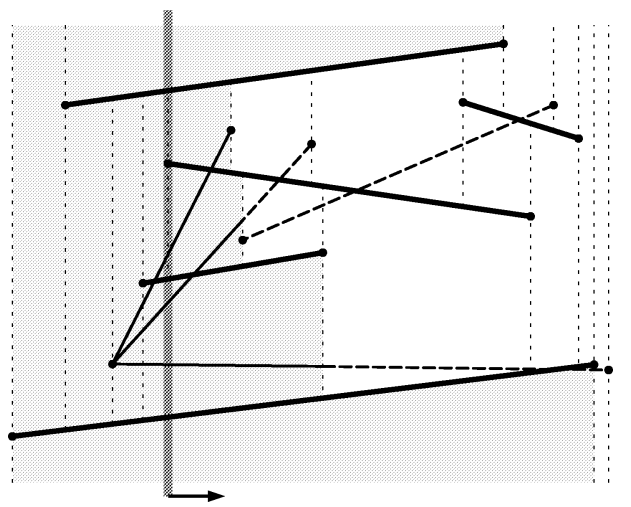

Fig. 7. Sweeping trapezoids; "blue" segments are darker.

As its data structures, Chan's algorithm needs only two ordered lists, one of trapezoids and one of red segments, ordered along sweep line $x=x_{s}$ or, equivalently, along the front. This makes his algorithm relatively easy to program, and causes it to perform considerably better than Bentley-Ottmann [4] or hereditary segment trees [21] on practical data [1]. It also works for curve segments where more than one intersection point is allowed, provided the endpoint/intersection order test has been implemented correctly.

\subsection{Eliminating the test for endpoint/intersection order}

With only above/below tests, it is impossible to determine where red segments enter and leave the trapezoids on the front. Fig. 8 shows a deformation of the four red curves from Fig. 7 that is consistent with the above/below tests on endpoints, even though the curves cross different sets of trapezoids. Once again, we must use endpoints as witnesses of intersection.

Conceptually deform the red curves, while respecting monotonicity and above/below tests, so that all intersections occur as far to the right as possible. This is how the deformation of Fig. 8 was chosen. Then sweep with a modified invariant: that intersections with a red segment whose witnesses are in or behind the sweep front have been reported, up to the first chance for the segment to leave the sweep front.

To maintain the invariant, our algorithm maintains the ordered list of blue segments that intersect the sweep line $x=x_{2}$, and, for each trapezoid on the front, a bundle of the deformed red segments that have entered that trapezoid from the left. The blue list supports logarithmic-time insertion and deletion. Bundles support logarithmic-time insertion, deletion, and binary search for a point on the sweep line (with constant time if the point is above or below all segments of the bundle) and split and merge in time proportional to the size of the bundle split off or merged, with a maximum of logarithmic time. Both lists and bundles can be implemented as standard balanced search trees.

Our algorithm maintains pointers between adjacent bundles and blue segments, and assumes that each red segment can discover its bundle in logarithmic time. In Chan's original algorithm, bundles and their pointers were located when needed by searching lists of red and blue segments; we find it easier to establish correctness for sweeping deformed red curves if the algorithm maintains bundles explicitly. 


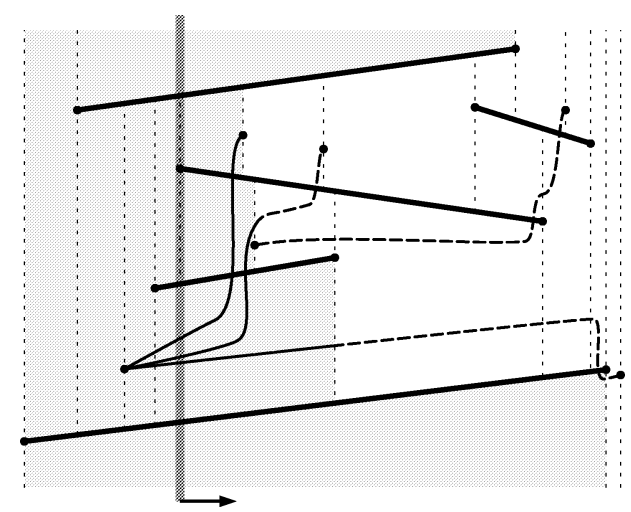

Fig. 8. Push intersections to right.

Theorem 8. The red/blue segment intersection problem for $x$-monotone pseudo-segments can be solved in optimal $\mathrm{O}(n \log n+k)$ time using endpoint $x$-order and above/below tests.

Proof. As noted in the previous section, work must be performed to re-establish the invariant when, and only when, the sweep line reaches a new trapezoid - that is, when it reaches an endpoint of a segment. We can assume, by using $y$ coordinates to break ties in $x$ coordinates, that if segments begin or end at the same $x$ coordinate, then they begin or end at the same point. Thus, we distinguish cases by the color of this endpoint.

Red endpoint $r$. At a red endpoint one trapezoid, $\tau$, ends and another, $\tau^{\prime}$, begins. Also, some red segments may end at $r$ and others may begin.

Let $r_{\mathrm{H}}$ and $r_{\mathrm{L}}$ denote the highest and lowest red segments ending at $r$. If the bundle for $r_{\mathrm{H}}$ is above $\tau$, then $r_{\mathrm{H}}$ and all segments between $r_{\mathrm{H}}$ and $\tau$ must be traced through blue segments below until they enter $\tau$. We can split the bundle of $r_{\mathrm{H}}$, collect all bundles between $r_{\mathrm{H}}$ and $\tau$, and merge into the bundle for $\tau$ - this work can be charged to red/blue intersections that are discovered. On the other hand, if the bundle for $r_{\mathrm{L}}$ is below $\tau$, then $r_{\mathrm{L}}$ and all segments between $r_{\mathrm{L}}$ and $\tau$ must be traced through blue segments above in a similar manner.

Finally, red segments ending at $r$ can be deleted, and those beginning at $r$ can be inserted. The total time for data structure manipulation is proportional to the number of intersections detected plus $\mathrm{O}(\log n)$ times the number of segments beginning and ending.

Blue endpoint $b$. At a blue endpoint where $i$ blue segments end and $j$ begin, we have $i+1$ trapezoids end (all but two of which are triangles) and $j+1$ trapezoids begin (again, all but two are triangles).

Let $\tau_{\mathrm{H}}$ denote the ending trapezoid whose upper right vertex is not $b$, and let $\tau_{\mathrm{L}}$ denote the ending trapezoid whose lower right vertex is not $b$. Note that $\tau_{\mathrm{H}}=\tau_{\mathrm{L}}$ if blue segments start, but do not end at $b$. By binary search on the bundles for all trapezoids, we can find which bundle to split by the point $b$, and then split it. If this bundle is below $\tau_{\mathrm{H}}$, then bundles between it and $\tau_{\mathrm{H}}$, inclusive, are merged into $\tau_{\mathrm{H}}$ and $\mathrm{red} / \mathrm{blue}$ intersections are reported. Similarly, if this bundle is below $\tau_{\mathrm{L}}$, then bundles are merged into $\tau_{\mathrm{L}}$ and intersections are reported.

Next, blue segments ending at $b$ are deleted and those starting at $b$ are inserted into the blue list. If no blue segment starts at $b$, the bundles for $\tau_{\mathrm{H}}$ and $\tau_{\mathrm{L}}$ are merged. 


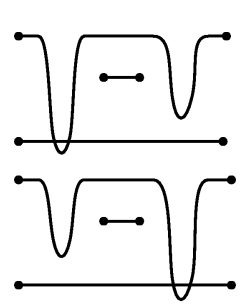

(a)

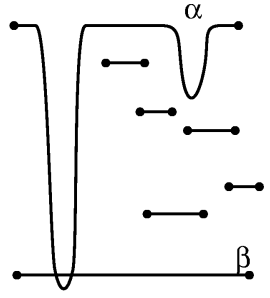

(b)

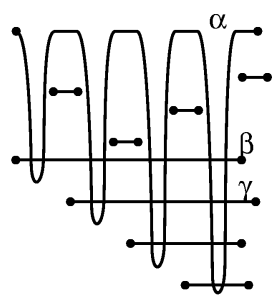

(c)

Fig. 9. No witness.

Searching, and splitting and merging bundles for $\tau_{\mathrm{H}}$ and $\tau_{\mathrm{L}}$ take $\mathrm{O}(\log n)$ time. All other splitting and merging can be charged to intersections reported.

By induction, we can show that the invariants are correctly maintained. The total time is $\mathrm{O}(n \log n+k)$.

\subsection{Difficulties with red/blue curves}

Since the modification above treats red segments as deformable curves, it should be no surprise that it works for pseudo-segments. When a pair of red and blue curves can intersect in more than one point, there are additional complications.

The primary difficulty is how to define a deformation of the red curves that moves intersection points to the right. Fig. 9(a) illustrates that with two crossings the intersection can appear before or after another curve's endpoints without changing above/below relationships. Since many short curves may come between two longer, intersecting curves as in Fig. 9(b), there is no simple witness that limits how far an intersection can move.

\section{Conclusions and open problems}

We have shown that endpoint orientation tests, above/below tests, and intersection tests are not sufficient to give efficient, output-sensitive algorithms for the general problem of curve segment intersection, although they are sufficient to give an $\mathrm{O}\left(n \log ^{2} n+k \log n\right)$ time algorithm for finding the $k$ intersections of $n$ line segments. In the red/blue case-where curves are colored red or blue and there are no red/red or blue/blue crossings - the $k$ intersections for $n$ line segments or $n$ curve pseudo-segments can be found in optimal $\mathrm{O}(n \log n+k)$ time.

Some open problems remain.

1. Is the logarithmic loss in efficiency necessary, or is there an optimal $\mathrm{O}(n \log n+k)$ algorithm for line segment intersection using degree 2 predicates?

2. Is there an algorithm that achieves $\Theta(n \sqrt{k})$ for curve segment intersection using only endpoint orientation, above/below, and intersection tests? Chan [11] has recently communicated an algorithm that achieves $\mathrm{O}(n \sqrt{k \log n})$.

3. Is there an efficient algorithm for red/blue curve segment intersections when pairs of curves may intersect in more than one point, using only endpoint orientation, above/below, and intersection tests? 


\section{Appendix A. Establishing degree bounds for curves}

In this appendix, we give the computations for the degree bounds listed in Table 2 for predicates for line segments and for the three example representations of monotone circular arcs from Section 2.3 that are illustrated in Fig. 2.

In all three representations of circular arcs, the $x$ coordinates of endpoints are represented in the input, so comparing the $x$-order of endpoints is a degree 1 predicate.

When a circle is represented by center $(a / 2, b / 2)$ and radius $r$, its standard equation is

$$
x^{2}+y^{2}=a x+b y+\left(r^{2}-\frac{a^{2}}{4}-\frac{b^{2}}{4}\right)=a x+b y-c .
$$

When a circle is represented by three points, $p=\left(x_{p}, y_{p}\right), q=\left(x_{q}, y_{q}\right)$ and $s=\left(x_{s}, y_{s}\right)$, the coefficients $a, b$ and $c$ are the solutions of the following system of three linear equations:

$$
\left(\begin{array}{ccc}
x_{p} & y_{p} & 1 \\
x_{q} & y_{q} & 1 \\
x_{s} & y_{s} & 1
\end{array}\right)\left(\begin{array}{c}
a \\
b \\
-c
\end{array}\right)=\left(\begin{array}{c}
x_{p}^{2}+y_{p}^{2} \\
x_{q}^{2}+y_{q}^{2} \\
x_{s}^{2}+y_{s}^{2}
\end{array}\right),
$$

and we have

$$
\begin{aligned}
& a=\frac{1}{D}\left|\begin{array}{lll}
x_{p}^{2}+y_{p}^{2} & y_{p} & 1 \\
x_{q}^{2}+y_{q}^{2} & y_{q} & 1 \\
x_{s}^{2}+y_{s}^{2} & y_{s} & 1
\end{array}\right|, \quad b=\frac{1}{D}\left|\begin{array}{ccc}
x_{p} & x_{p}^{2}+y_{p}^{2} & 1 \\
x_{q} & x_{q}^{2}+y_{q}^{2} & 1 \\
x_{s} & x_{s}^{2}+y_{s}^{2} & 1
\end{array}\right|, \quad c=-\frac{1}{D}\left|\begin{array}{ccc}
x_{p} & y_{p} & x_{p}^{2}+y_{p}^{2} \\
x_{q} & y_{q} & x_{q}^{2}+y_{q}^{2} \\
x_{s} & y_{s} & x_{s}^{2}+y_{s}^{2}
\end{array}\right| \\
& \text { with } D=\left|\begin{array}{lll}
x_{p} & y_{p} & 1 \\
x_{q} & y_{q} & 1 \\
x_{s} & y_{s} & 1
\end{array}\right| .
\end{aligned}
$$

It follows from Eqs. (A.1) and (A.2) that the incircle predicate that decides whether a point lies inside a circle has degree 2 when the circle is defined by its center and its radius, and degree 4 if it is defined by three points.

To decide if a point $A$ is above or below an $\operatorname{arc} \gamma$, we first test if the point lies inside the circle $\Gamma$ supporting $\gamma$. If it does, then we can determine above or below, otherwise we compare the $y$-coordinates of $A$ and the center of $\Gamma$. Thus, the degree of this predicate is again 2 when the circle $\Gamma$ is defined by its center and its radius, and 4 if it is defined by three points.

Consider now checking if two circles $C_{1}$ and $C_{2}$ intersect. When the circles are defined by centers and radii, this reduces to comparing the distance between the centers and the sum and the difference of the radii, which is clearly a degree 2 computation. Let us consider the case of circles defined by three points. Subtracting the equations for two circles in standard form gives a line, called the chordal line of the two circles, whose equation is

$$
\left(b_{1}-b_{2}\right) y=-\left(a_{1}-a_{2}\right) x+c_{1}-c_{2} .
$$

Using Eq. (A.3), we can eliminate the $y$ variable in one of the equations of the circles (A.1). We then obtain a univariate polynomial of degree 2 in $x$, namely $P(x)=A x^{2}+B x+C$, where 


$$
\begin{aligned}
& A=\left(a_{1}-a_{2}\right)^{2}+\left(b_{1}-b_{2}\right)^{2}, \\
& B=\left(b_{1}-b_{2}\right)\left(a_{1} b_{2}-a_{2} b_{1}\right)-2\left(a_{1}-a_{2}\right)\left(c_{1}-c_{2}\right), \\
& C=\left(c_{1}-c_{2}\right)^{2}+\left(b_{1}-b_{2}\right)\left(b_{1} c_{2}-b_{2} c_{1}\right) .
\end{aligned}
$$

Observe that, when circles are represented by center and radius, $P(x)$ has integral coefficients $A, B$ and $C$ of degrees respectively 2, 3 and 4 . When circles are represented by three points, the degrees of the numerators of $A, B$ and $C$ become 10, 11 and 12, respectively. The denominators are identical and of degree 8 .

The two circles intersect if and only if the discriminant $\Delta=B^{2}-4 A C$ of $P(x)$ is positive. Easy computations show that

$$
\Delta=\left(b_{1}-b_{2}\right)^{2}\left(\left(a_{1} b_{2}-a_{2} b_{1}\right)^{2}-4\left(a_{1}-a_{2}\right)\left(a_{1} c_{2}-a_{2} c_{1}\right)-4\left(b_{1}-b_{2}\right)\left(b_{1} c_{2}-b_{2} c_{1}\right)-4\left(c_{1}-c_{2}\right)^{2}\right) .
$$

It follows that evaluating the sign of $\Delta$ is a degree 12 computation when the circles are defined by three points.

Consider now checking if two (monotone) circle segments $\gamma_{1}$ and $\gamma_{2}$ intersect, which can only happen if the supporting circles $\Gamma_{1}$ and $\Gamma_{2}$ intersect. Let $I$ and $J$ be the intersection points of those circles, with $I$ lying left of $J$. Let points $A_{1}$ and $B_{1}$ be the endpoints of $\gamma_{1}$ from left to right. If $A_{1}$ and $B_{1}$ lie on opposite sides of the chordal line $H$ of $\Gamma_{1}$ and $\Gamma_{2}$, then $\gamma_{1}$ contains either $I$ or $J$. (Specifically, when $\Gamma_{1}$ is an upper arc, if $A_{1}$ is above $H$ then, $\gamma_{1}$ contains $I$, and if $A_{1}$ is below $H$ then $\gamma_{1}$ contains $J$.) Otherwise, $\gamma_{1}$ contains both $I$ and $J$ or none. To distinguish between these cases, we can either check whether the endpoints $A_{1}$ and $B_{1}$ of $\gamma_{1}$ lie on opposite sides of the line passing through the centers of the circles (a degree 2 computation when the circles are defined by centers and radii) or check whether the midpoint $M$ of segment $I J$ is right of $A_{1}$ and left of $B_{1}$ (since the abscissa of $M$ is equal to $-B / 2 A$, this is a degree 11 computation when the circles are defined by three points). Because the same discussion can be applied to $\gamma_{2}$, we conclude that the degree of the intersection predicate for circle segments is the same as the degree of the intersection predicate for circles.

We next evaluate the degrees of the predicates for endpoint/intersection order and intersection $x$-order. Consider two intersecting circles. The roots of $P(x)=0$ are the $x$-coordinates of the intersection points of the circles. It then follows that sorting the $x$-coordinate $x_{E}$ of an endpoint with respect to the $x$-coordinates of the intersection points reduces to evaluating the signs of $\left(A x_{E}^{2}+B x_{E}+C\right)$ and $\left(2 A x_{E}+B\right)$. Sorting the $x$-coordinates of the intersection points of two pairs of circles reduces to evaluating the sign of

$$
A\left(-B^{\prime}+\sqrt{B^{\prime 2}-4 A^{\prime} C^{\prime}}\right)-A^{\prime}\left(-B+\sqrt{B^{2}-4 A C}\right) .
$$

By squaring twice, it can be seen that this reduces to evaluating the sign of

$$
\left(\left(A B^{\prime}-A^{\prime} B\right)^{2}-A^{2}\left(B^{\prime 2}-4 A^{\prime} C^{\prime}\right)-A^{\prime 2}\left(B^{2}-4 A C\right)\right)^{2}-4 A^{2} A^{\prime 2}\left(B^{2}-4 A C\right)\left(B^{\prime 2}-4 A^{\prime} C^{\prime}\right),
$$

which can be rewritten as

$$
16 A^{2} A^{\prime 2}\left(\left(A C^{\prime}-A^{\prime} C\right)^{2}-\left(A B^{\prime}-A^{\prime} B\right)\left(B C^{\prime}-B^{\prime} C\right)\right) \text {. }
$$

When circles are represented by center and radius, we have seen that the degrees of $A, B$ and $C$ are respectively 2, 3 and 4 . It follows that the degree of the endpoint/intersection predicate is 4 and that the degree of the intersection/intersection predicate is 12 . When circles are represented by three points, the degrees of the numerators of $A, B$ and $C$ become 10, 11 and 12, respectively, and they all have the 
same denominator. It follows that the degree of the endpoint/intersection and intersection/intersection predicates are 12 and 44.

To order the intersections along one circle segment, we decide whether the two corresponding intersection lines cross inside the circle or outside. This takes degree 6 or degree 16.

\section{References}

[1] D.S. Andrews, J. Snoeyink, J. Boritz, T. Chan, G. Denham, J. Harrison, C. Zhu, Further comparison of algorithms for geometric intersection problems, in: Proc. 6th Internat. Sympos. Spatial Data Handling, 1994, pp. 709-724.

[2] F. Avnaim, J.-D. Boissonnat, O. Devillers, F.P. Preparata, M. Yvinec, Evaluating signs of determinants using single-precision arithmetic, Algorithmica 17 (2) (1997) 111-132.

[3] I.J. Balaban, An optimal algorithm for finding segment intersections, in: Proc. 11th Annu. ACM Sympos. Comput. Geom., 1995, pp. 211-219.

[4] J.L. Bentley, T.A. Ottmann, Algorithms for reporting and counting geometric intersections, IEEE Trans. Comput. 28 (9) (1979) 643-647.

[5] J.-D. Boissonnat and F.P. Preparata, Robust plane sweep for intersecting segments, Technical Report RR-3270, INRIA Sophia Antipolis, September 1997. To appear in SIAM J. Comput. //www.inria.fr:80/ RRRT/publications-eng.html.

[6] H. Brönnimann, C. Burnikel, S. Pion, Interval arithmetic yields efficient dynamic filters for computational geometry, in: Proc. 14th Annu. ACM Sympos. Comput. Geom., 1998, pp. 165-174.

[7] H. Brönnimann, I. Emiris, V. Pan, S. Pion, Sign determination in Residue Number Systems, Theoret. Comput. Sci. 210 (1) (1999) 173-197. Special Issue on Real Numbers and Computers.

[8] C. Burnikel, Exact computation of Voronoi diagrams and line segment intersections, Ph.D. Thesis, Universität des Saarlandes, March 1996.

[9] C. Burnikel, J. Könnemann, K. Mehlhorn, S. Näher, S. Schirra, C. Uhrig, Exact geometric computation in LEDA, in: Proc. 11th Annu. ACM Sympos. Comput. Geom., 1995, pp. C18-C19.

[10] T.M. Chan, A simple trapezoid sweep algorithm for reporting red/blue segment intersections, in: Proc. 6th Canad. Conf. Comput. Geom., 1994, pp. 263-268.

[11] T.M.Y. Chan, Private communication, August 1998.

[12] B. Chazelle, H. Edelsbrunner, An optimal algorithm for intersecting line segments in the plane, J. ACM 39 (1) (1992) 1-54.

[13] K.L. Clarkson, Safe and effective determinant evaluation, in: Proc. 33rd Annu. IEEE Sympos. Found. Comput. Sci., 1992, pp. 387-395.

[14] A.R. Forrest, Invited talk on computational geometry and software engineering, in: 2nd Annu. ACM Sympos. Comput. Geom., 1986.

[15] S. Fortune, C.J. Van Wyk, Static analysis yields efficient exact integer arithmetic for computational geometry, ACM Trans. Graph. 15 (3) (1996) 223-248.

[16] L.J. Guibas, D. Salesin, J. Stolfi, Epsilon geometry: building robust algorithms from imprecise computations, in: Proc. 5th Annu. ACM Sympos. Comput. Geom., 1989, pp. 208-217.

[17] J. Hershberger, S. Suri, Applications of a semi-dynamic convex hull algorithm, BIT 32 (1992) 249-267.

[18] D.T. Lee, F.P. Preparata, Computational geometry: a survey, IEEE Trans. Comput. 33 (1984) 1072-1101.

[19] G. Liotta, F.P. Preparata, R. Tamassia, Robust proximity queries: an illustration of degree-driven algorithm design, in: Proc. 13th Annu. ACM Sympos. Comput. Geom., 1997, pp. 156-165.

[20] V.J. Milenkovic, Verifiable implementations of geometric algorithms using finite precision arithmetic, Ph.D. Thesis, Carnegie-Mellon University, Pittsburg, PA, 1988. 
[21] L. Palazzi, J. Snoeyink, Counting and reporting red/blue segment intersections, CVGIP: Graph. Models Image Process. 56 (4) (1994) 304-311.

[22] F.P. Preparata, M.I. Shamos, Computational Geometry: An Introduction, Springer, New York, 1985.

[23] D. Priest, On properties of floating point arithmetics: numerical stability and the cost of accurate computations, Ph.D. Thesis, Department of Mathematics, University of California at Berkeley, 1992.

[24] J.R. Shewchuk, Robust adaptive floating-point geometric predicates, in: Proc. 12th Annu. ACM Sympos. Comput. Geom., 1996, pp. 141-150.

[25] K. Sugihara, M. Iri, A robust topology-oriented incremental algorithm for Voronoi diagrams, Internat. J. Comput. Geom. Appl. 4 (2) (1994) 179-228.

[26] C. Yap, Towards exact geometric computation, Computational Geometry 7 (1-2) (1997) 3-23. 